\title{
Evaluation of a measles vaccine campaign by oral-fluid surveys in a rural Kenyan district: interpretation of antibody prevalence data using mixture models
}

E. O. OHUMA ${ }^{1 *}$, E. A. OKIRO ${ }^{1}$, A. BETT ${ }^{1}$, J. ABWAO ${ }^{1}$, S. WERE ${ }^{2}$, D. SAMUEL ${ }^{3}$, A. VYSE ${ }^{4}$, N. GAY ${ }^{4}$, D. W. G. BROWN ${ }^{3}$ AND D. J. NOKES $S^{1,5}$

${ }^{1}$ Centre for Geographic Medicine Research - Coast, Kenya Medical Research Institute, Kilifi, Kenya

${ }^{2}$ Kilifi District Hospital, Kenya

${ }^{3}$ Virus Reference Department, Centre for Infections, Health Protection Agency, London, UK

${ }^{4}$ Modelling and Economics Unit, Centre for Infections, Health Protection Agency, London, UK

${ }^{5}$ Department of Biological Sciences, University of Warwick, Coventry, UK

(Accepted 9 May 2008; first published online 10 June 2008)

\section{SUMMARY}

We evaluated the effectiveness of a measles vaccine campaign in rural Kenya, based on oral-fluid surveys and mixture-modelling analysis. Specimens were collected from 886 children aged 9 months to 14 years pre-campaign and from a comparison sample of 598 children aged 6 months post-campaign. Quantitative measles-specific antibody data were obtained by commercial kit. The estimated proportions of measles-specific antibody negative in children aged 0-4, 5-9 and $10-14$ years were $51 \%, 42 \%$ and $27 \%$, respectively, pre- campaign and $18 \%, 14 \%$ and $6 \%$, respectively, post-campaign. We estimate a reduction in the proportion susceptible of $65-78 \%$, with $\sim 85 \%$ of the population recorded to have received vaccine. The proportion of 'weak' positive individuals rose from $35 \%$ pre-campaign to $54 \%$ post-campaign. Our results confirm the effectiveness of the campaign in reducing susceptibility to measles and demonstrate the potential of oral-fluid studies to monitor the impact of measles vaccination campaigns.

Key words: Campaign vaccination, measles antibodies, mixture modelling, oral fluid, Kenya.

\section{INTRODUCTION}

There is now a body of evidence supporting the utility of minimally invasive oral-fluid collection for immune status determination [1-7]. Technical difficulties in the sensitivity of assays for antibody detection in oral fluid have been addressed [1,8] and oral-fluid antibody surveys have shown excellent potential as a suitable alternative to blood collection especially in

\footnotetext{
* Author for correspondence: Mr E. O. Ohuma, Kenya Medical Research Institute (KEMRI)/Wellcome Trust Research Programme, Centre for Geographic Medicine Research (Coast), PO Box 230, 80108, Kilifi, Kenya.

(Email: eohuma@kilifi.kemri-wellcome.org)
}

evaluating population immunity prevalence $[1,3,8]$. Wider implementation requires demonstration of a useful role in vaccine programme development and refinement, for example, in evaluating the effectiveness of immunization campaigns. Commercial assays are now available that afford an easy and standardized approach to anti-measles-specific IgG antibody testing in oral-fluid surveys [2,9]. However, a remaining concern is the performance of oral-fluid antibody assays in differing settings, for example, populations with high levels of vaccine-induced immunity with consequent low-level specific antibody [2, 10-12].

We undertook an analysis of unpublished data collected at the time of and after a national measles 
vaccination campaign targeting children aged 9 months to 14 years, in a rural district of Kenya in 2002. This campaign formed part of a national accelerated measles control initiative which began in 2002 [13]. The data was analysed using mixture modelling as previously applied successfully to oralfluid prevalence data for rubella $[1,8]$. The objective was to assess the use of oral-fluid surveys as a means of defining population antibody prevalence, assessing the impact of a mass campaign and estimating the level of susceptibility in the vaccine recipients. This is the first time mixture modelling has been applied in the interpretation of oral-fluid data for measles.

\section{METHODS}

\section{Sampling}

The 2002 campaign evaluation was undertaken in Kilifi District, coastal Kenya, which comprises a predominantly rural farming population of around 545000 [14]. Kilifi town, with around 30000 occupants, is the location of the Kilifi District hospital (KDH). Official statistics (2002) on routine vaccine uptake for measles in Kilifi District reported a coverage of $72 \%$ [13]. A measles vaccine campaign was carried out over the period 17-23 June 2002, following an operation of public awareness (Ministry of Health, personal communication). Campaign vaccination sites included Government health facilities and private clinics, targeting children aged 9 months to 6 years, and schools, targeting children aged between 5 and 14 years. The study was undertaken in cooperation with the local Ministry of Health and Kenyan Expanded Programme on Immunization (KEPI). Ethical approval for the study was granted by the Kenya Medical Research Institute (KEMRI)/ National Ethical Review Committee, in Kenya and Coventry Research Ethics Committee, in the United Kingdom.

The study sampling design was intended to estimate antibody prevalence representative of contrasting sections of the population (rural vs. town), at two timepoints: (i) at the moment of the campaign in order to assess pre-campaign levels of measles antibody prevalence in vaccine recipients (17-23 June 2002), and (ii) $>1$ month post-campaign (11 November-20 December 2002), to assess population seroprevalence influenced by campaign vaccination. Two locations from a total of 15 in the district were chosen, namely, Kilifi township (the town and its immediate surroun- dings), and Ngerenya, a rural community situated $13 \mathrm{~km}$ north of Kilifi town. The pre-campaign survey consisted of the selection of the main health facility (Maternal Child Health Clinic at KDH and Ngerenya Health Centre) and the three largest schools in each of the two locations. The study proceeded after consultation with the head teacher or senior nurse and their staff in the schools and clinics respectively and thereafter study information sheets were provided for participants. At each health facility, children were selected on an ad hoc basis up to a maximum of 35 $(\mathrm{KDH})$ or, due to a slower recruitment rate, 25 (Ngerenya), for each age group 9-11, 12-23, 24-35, 36-47, 48-59, and 60-71 months. Within the schools, samples of 10 children for each yearly age group from 5-14 years were selected as they arrived for vaccination. All participating children were requested to provide an oral-fluid sample, and data were collected on previous routine measles vaccination.

For the post-campaign survey, the sampling frame was the total population of children who, at the time of the campaign, were aged between 9 months and 14 years within each of the two locations. Children numbering 100 in each of the age groups: 9 months -4 years, 5-9 years and 10-14 years were selected by pure random sampling from the register of the demographic surveillance system (DSS) established by KEMRI/Wellcome Trust Research Programme. Local chiefs were consulted in advance of the study and information disseminated through meetings of elders. Field teams visited the family of each child to invite them to participate in the study, and from those who consented, an oral-fluid sample was requested, and data collected on whether the child received measles vaccine during the campaign. For those who were aged $<5$ years, information on previous routine measles vaccination uptake was also obtained. Whenever the participation of a selected child was declined, a replacement was selected at random from the census register. For any child identified as having not been vaccinated against measles, the mother was encouraged to take the child to the nearest vaccination centre.

\section{Laboratory methods}

Oral-fluid samples were collected by Oracol device (Malvern Medical Developments, Worcester, UK), using the method previously described [2, 5]. All specimens were labelled with date of sample collection, initials of child, and a code comprising sample 
number for specific age group from specified school/ clinic. Collected samples were stored in vaccine boxes with ice packs and transferred at the end of each working day to the laboratory at $\mathrm{KDH}$. Oral fluids were processed as previously described and stored at $-80{ }^{\circ} \mathrm{C}[2,5]$, and screened for measles-specific antibody using the Microimmune measles IgG EIA (Microimmune Ltd, Middlesex, UK) according to the manufacturer's instructions. This assay was developed for use with both oral-fluid and serum specimens, and has an antibody capture format which has been shown to perform well for specimens with low-level specific antibody [7,9]. The antibody measured in the Microimmune assay is specific to the measles nucleoprotein and its detection interpreted as an indicator of exposure to virus (via infection or vaccination) rather than a measure of protection.

\section{Data analysis}

The proportion seropositive and seronegative for measles-specific $\operatorname{IgG}$ antibodies was estimated using mixture modelling, as previously described for the analysis of rubella-specific IgG data from oral-fluid samples by Gay et al. [1], in order to overcome the imperfect sensitivity usually associated with oral-fluid assays. The Microimmune assay data are recorded as optical density (OD) $\mathrm{T} / \mathrm{N}$ ratios [test $(\mathrm{T})$, sample OD reading divided by the average OD readings of three negative $(\mathrm{N})$, kit controls] for the pre-campaign and post-campaign. The $\mathrm{T} / \mathrm{N}$ ratios are log-transformed to base $10\left(\log _{10}\right)$ for normality and further aggregated into reactivity categories of equal-width bands on the $\log _{10}$ scale.

Each individual's specific antibody level $\left[\log _{10}(\mathrm{~T} /\right.$ $\mathrm{N})$ ] is assumed to fall into one of three status categories: strong positive, weak positive or negative. The rationale for including a weak positive group was to account for possible waning antibody levels with increasing age, account for lower specific antibody levels induced by vaccination compared to those with wild-type infection and low concentration of specific $\mathrm{IgG}$ often found in oral fluids at the very limit of detection of the assay systems [12, 15-17].

We further assume that the distribution of the antibody (reactivity) levels in each status category is independent of age and follows a normal distribution and that it is only the proportions in each age group that vary by status and reactivity category. We fit three distributions representing each status and compute the mean and standard deviation of each. The same model structure and parameters (mean and standard deviation) are maintained during the precampaign and post-campaign thus enabling the results of both the pre- and post-campaign to be directly compared and appropriate conclusions drawn.

We therefore define a model comprising $K=3$ components (status categories), where $f_{i}(x)$ denotes the distribution of reactivity levels $(x)$ for the $i$ th component and $p_{i j}$ denotes the proportion of samples from the $i$ th component in age group $j$. Then the overall density of reactivity levels, at age group $j$, $F_{j}(x)$, is a mixture of the component densities described as follows

$F_{j}(x)=\sum_{i=1}^{K} p_{i j} f_{i}(x)$.

Using 5-year age groups (0-4 years, 5-9 years and 10-14 years), a total of 18 parameters were estimated; 12 describing the proportions negative and weak positive (with the proportion strong positive defined as the remainder making unity) in the three age groups for both pre- and post-campaign and six describing the mean and standard deviation of the three statuses. We also analysed the data in single-year age groups estimating 66 parameters (60 describing the proportions in each age for both pre- and postcampaign and six describing the mean and standard deviation of the three status distributions). Maximum-likelihood estimates for the model's parameters were estimated by optimization achieved by minimizing the deviance, $D$, using the Microsoft Excel 97 Solver Add-In routine (Microsoft Corporation, USA) where $D=2\left(\mathrm{LL}_{\mathrm{S}}-\mathrm{LL}_{\mathrm{R}}\right)$, where $\mathrm{R}$ is the researcher's model, $\mathrm{S}$ the saturated model, and LL the log-likelihood. Likelihood-based $95 \%$ confidence intervals (CIs) for the age-specific prevalence were obtained by finding the maximum and minimum values for which the deviance was within $3.84(95 \%$ $\mathrm{CI}$ of the $\chi^{2}$ distribution) of the minimum. Full details of the method of data preparation, mixture model derivation, and estimation of parameters with $95 \%$ CIs have been published [1, 8]. The assertion that a three-distribution mixture model for antibody levels (i.e. a population of negative, weak positives and strong positives) provided a better fit to the data than a two-distribution model (i.e. positives and negatives), was assessed using the likelihood ratio test, i.e. the difference in deviance $D$ for the two models was assessed for significance assuming a $\chi^{2}$ distribution with 
degrees of freedom equal to the difference in number of parameters for the two models.

\section{RESULTS}

In the pre-campaign survey 886 children were interviewed, 488 (49\% males) from Kilifi town ( $38 \%$ from $\mathrm{KDH}$, and $20-21 \%$ from each of the three schools), and 398 (47\% males) from the rural Ngerenya location (23\% from the clinic and $25-26 \%$ from each of the three schools). In the post-campaign survey 598 children were interviewed, 294 ( $52 \%$ males) from Kilifi town and 304 (47\% males) from Ngerenya. For each of the age groups 9 months -4 years, 5-9 years and 10-14 years, the numbers of children providing oral-fluid samples pre-vaccination, were 194, 347 and 325. For the corresponding age groups postcampaign, the numbers sampled were 158, 195 and 201 , respectively.

Results from the post-vaccination questionnaire survey recorded $85 \%(450 / 531)$ of the population as having received the vaccine during the campaign, with both locations depicting the same coverage in terms of the proportion vaccinated. In total, $86 \%(241 / 280)$ of females were vaccinated compared to $83 \%(209 /$ 251 ) of males. The percentage vaccinated was $76 \%$, $93 \%$ and $85 \%$ in the age groups $0-4,5-9$ and $10-14$ years, respectively, and these did not differ significantly in the two locations. Verification of previous routine measles vaccination in children aged $<5$ years was mostly by parental recall, 93/153 (history, $61 \%$ ), compared to $50 / 153(33 \%)$ confirmed through immunization cards. A relatively higher percentage had card-confirmed routine vaccination in Kilifi township, $31 / 80(39 \%)$ by card and $44 / 80(55 \%)$ by parental recall, compared to the rural location of Ngerenya, $22 / 85(26 \%)$ and 58/85 (68\%), respectively.

A comparison of the frequency distribution of quantitative data pre- and post-campaign showed a marked difference for each of the three age groups (Fig. $1 a-c$ ). The post- vaccination results showed a more marked skew ('shift') to the right indicating that this population consists of samples that gave stronger readings in the assay and therefore contain more measles-specific $\mathrm{IgG}$ positives. The distribution of reactivity categories by status (negative, weak positive and strong positive) are presented in Figure $1 d$. The three-distribution (negative, weak positive and strong positive) mixture model provided a significantly improved fit to the data (deviance $=129 \cdot 104$ on 108 D.F.) compared to the two-distribution (negative and
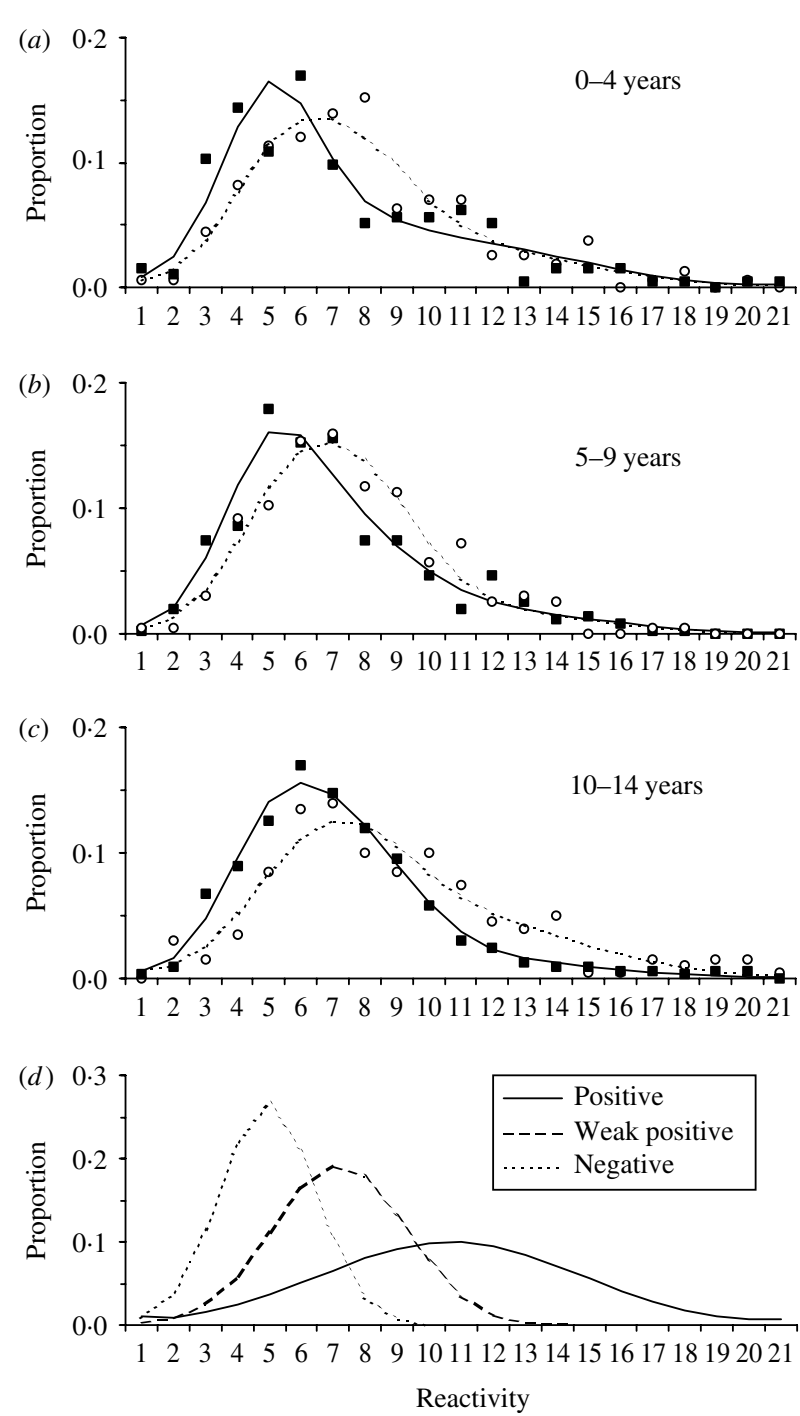

Fig. 1. Resultant fit of a mixture model applied to measlesspecific antibody data from surveys pre- and post-vaccination campaign in Kilifi District, Kenya, 2002. $(a-c)$ Frequency in percentage by antibody reactivity category [with equal-width bands based on $\log$ OD T/N values - see main text for explanation)] for pre-campaign raw data ( $(\mathbf{C})$ and model fit $(-)$ and post-campaign raw data $(\bigcirc)$ and model fit (- - - ) for age groups (a) 0-4 years, (b) 5-9 years, (c) 10-14 years. (d) The modelled distributions of the positive, weak positive and negative components.

positive) mixture model (deviance $=171.996$ on 116 D.F., i.e. difference in deviance $42.892, P$ value $<0.001)$.

Overall measles antibody prevalence in the target age-group of children aged 9 months-14 years was estimated to be $60 \%$ pre-campaign, and $87 \%$ postcampaign. This represents a reduction in susceptibility prevalence of $70 \%$. Age-specific reductions in seronegative prevalence (shown in Fig. 2) are $65 \%, 67 \%$, 

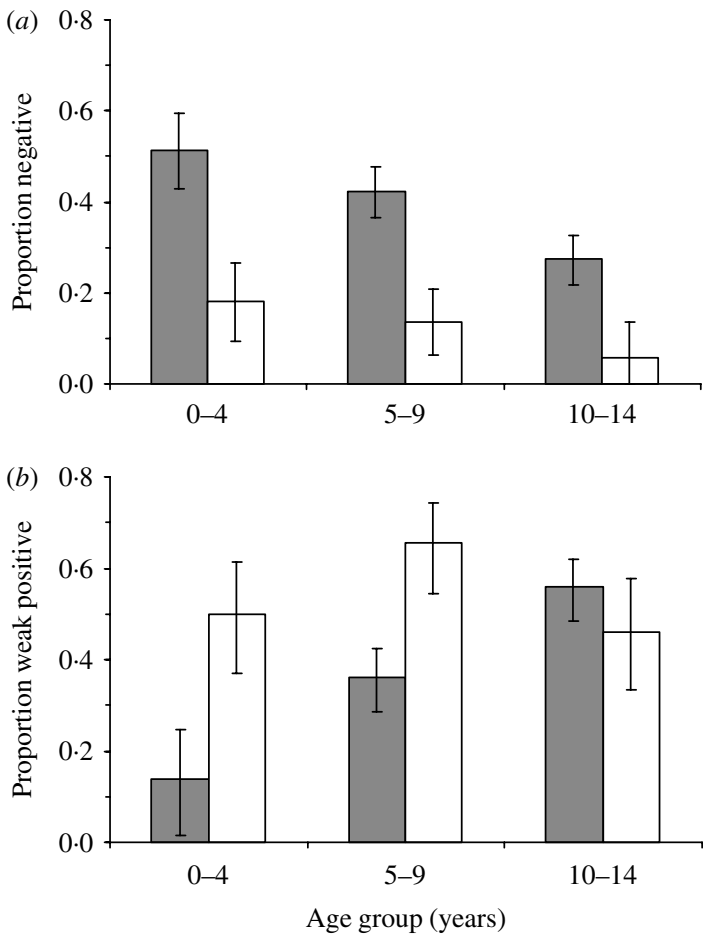

Fig. 2. Comparison of model estimates of $(a)$ measles seronegativity and $(b)$ proportion weakly positive by age group in children pre-campaign ( $\square$ ) and post-campaign $(\square)$ in Kilifi District, Kenya, 2002 (95\% confidence intervals are shown).

and $78 \%$ in age groups 9 months -4 years, $5-9$ years and $10-14$ years, respectively. However, there remains a high proportion (11\%), antibody negative in 14year-olds despite the intense campaign outreach and vaccination. The prevalence of weak-positive individuals is $54 \%$ post-campaign relative to $35 \%$ pre-campaign, with increases (which are statistically significant) restricted to the 9 months -9 years age groups (Fig. 2b).

Differences by location were also noted with $29 \%$ seropositive in Ngerenya (rural) compared to $63 \%$ for Kilifi township pre-vaccination in children aged $<5$ years (Fig. 3), but not in older ages. However, post-campaign, this prevalence differential between the two locations in young children was no longer evident. The proportion of children aged $<5$ years who reported routine measles vaccination (by card or recall) was $83 \%$ of 64 children in Ngerenya and $97 \%$ of 148 children in Kilifi.

\section{DISCUSSION}

A key objective of the present study was to provide an independent evaluation of the effectiveness of a

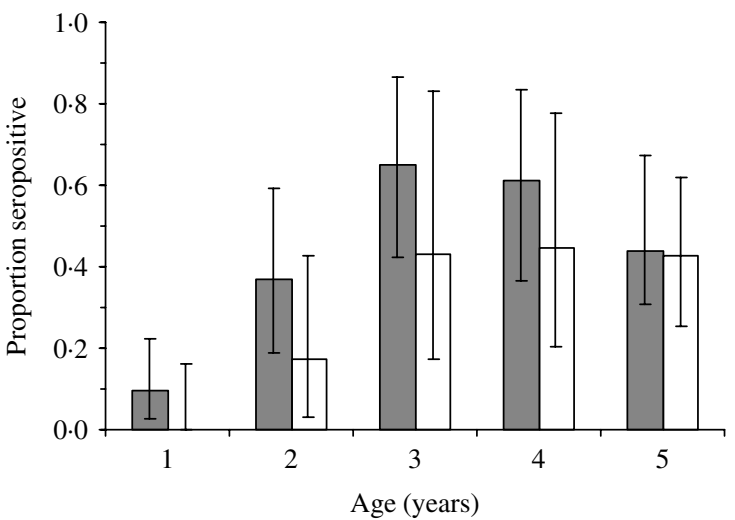

Fig. 3. Comparison of model estimates of measles seropositivity by age in children aged $1-5$ years residing in Kilifi township ( $\square$ ) and in a rural area, Ngerenya ( $\square$ ), precampaign, Kilifi District, Kenya, 2002.

measles vaccination campaign undertaken in a typical predominantly rural developing country setting using oral-fluid surveys. Estimates of seroprevalence prior to and after the campaign were obtained by application of the method of mixture modelling on specific measles antibody data, and based on a similar analysis performed on rubella antibodies derived from oralfluid sampling in Ethiopia [1]. It has previously been shown that a fixed cut-off method of determining measles immune status is problematical in populations with high vaccine coverage, in which specific measles antibody levels have been shown to be relatively low, with concomitant problems in assay sensitivity $[11,12]$. In this study, we demonstrate the usefulness of the mixture-modelling approach for data in which the distributions of antibody levels for negatives and positives are not easily distinguishable. This is the first example of such an application to measles serological data.

Our study clearly depicts a considerable impact on the population measles seropositive prevalence - assumed to reflect immunity - resulting from the campaign. We record an overall reduction in seronegative prevalence of $\sim 70 \%$ (range $65-78 \%$ ). Our questionnaire data suggests around $85 \%$ of the population received vaccine during the campaign, which is not inconsistent with the results of a sampling survey of supplementary immunization activities (SIA) coverage for Coast Province in which Kilifi resides [13]. If $85 \%$ of susceptible children were vaccinated, and assuming a vaccine take of $90 \%$, the expected reduction in the 'susceptible' proportion is $76.5 \%$ $(90 \% \times 85 \%)$, an estimate consistent with the upper limit of the estimate from the mixture model. A smaller 
reduction in susceptibility would be expected if 'hard to reach' children were missed by both routine vaccination and the vaccination campaign [18]. The 70\% reduction shown by our survey data suggests that the vaccination campaign was largely successful at reaching previously unvaccinated children. However, approximately $18 \%$ of $<5$-year-olds still remain susceptible to measles even after the vaccination campaign hence posing some risk of measles spread. Furthermore, although the proportion that are seronegative decrease as age increases, there is a significant proportion seronegative especially in the 14 years age group. An additional concern is the low seroprevalence of antibody prior to the campaign, suggesting that routine vaccination coverage is low and that susceptibles will rapidly re-accumulate. It is thus clear that routine and supplementary immunization and campaign activities need to be strengthened to increase population immunity and in order to establish protective herd immunity.

The optimal study design by which to assess the effectiveness of the vaccination campaign would have been a random sample selected from the DSS register prior to the campaign with follow-up sampling of the same individuals 1 month after the campaign. As a pragmatic response to the short notice given for the campaign we undertook purposive sampling from individuals attending for vaccination as a substitute for the pre-campaign randomized survey, and the postcampaign sample was delayed for 5 months awaiting official clearance. Although the extent of the influence of this sampling design on the results is unclear, we suspect it would not have markedly altered the outcome since we know from the post-campaign survey that the vast majority ( $\sim 85 \%$ ) of eligible individuals attended for campaign vaccination (Department of Health estimates were $90 \%$ for the Kenyan coast) and there was reportedly no instance of measles in the district in the 6 months post-campaign.

In our study the reduction in the proportion seronegative following the vaccine campaign was greater in the rural location, since the pre-vaccination prevalence of measles-specific antibodies was markedly lower than in the town location in children aged $<5$ years. Proportions positive in the rural and town populations seems to have risen considerably after the campaign with the rural population rising to relatively higher levels than the urban population primarily due to the fact that there were more susceptibles residing in the rural areas that had not been previously vaccinated. The rural population is more at risk compared to the urban as most of them appear not to seek early immunization and these differences could possibly be attributable to proximity, accessibility (to hospital and health centres) and lack of information among the rural population.

The observed increase in high proportions of individuals with low positive antibody levels ('weak positives') post-campaign ( $54 \%$ ) compared to precampaign (35\%) is not unexpected where specific antibodies are the result of vaccination [17]. Interestingly, this increase is not identified in the 10-14 years age group, suggesting an age-dependence in the antibody response following vaccination. The role of weakly positive individuals as a potential source for continued measles transmission, although unlikely, cannot be completely discounted. Further studies are required to establish the relationship between lowlevel antibody identified by mixture-modelling techniques and susceptibility to infection, and relate this to similar work for fixed cut-offs (see e.g. [12]). This group of individuals may become significant when very high levels of vaccination arise, resulting in little wild-type infection, where most immunity is vaccine induced and correspondingly of low titre, and where boosting of antibody titres also cannot occur $[12,17]$.

Data on measles surveillance shows little measles transmission 4 years post-vaccination, indicative of an effective campaign with good vaccine efficacy [13]. However, an epidemic arose in May 2006 to March 2007 with (196 hospital cases reported, 36/ 100000 in Kilifi District) most of the reported cases ( $77 \%$ ) occurring in early life ( $<5$ years), with $35 \%$ aged $<9$ months, $18 \%$ (9-23 months) and $24 \%$ (24-59 months), compared to $23 \%$ occurring in children aged $>5$ years, with $17 \%$ (5-9 years) and 6\% (10-12 years). Ironically, a SIA scheduled for July 2005 approximately 36 months after the catch-up SIA of 2002 [19] was postponed, and implemented in July 2006. This delay was probably a contributing factor in bringing about the outbreak, and serves to emphasize the importance of increasing the levels of routine measles vaccine uptake and the need for timely supplementary immunization activities as suggested by the WHO Regional Office for Africa reports $[13,19,20]$.

\section{ACKNOWLEDGEMENTS}

We thank the Ministry of Health, Kilifi District Hospital, Ngerenya Health Centre, the schools within the two areas, and Kilifi administration offices for 
their support; thanks are also due to the study participants. We acknowledge all participants, surveillance teams and medical personnel for their contribution to this work. The research is published with the permission of the Director of KEMRI. The work was supported by the Wellcome Trust (ref. 061584).

\section{DECLARATION OF INTEREST}

Dr Dhan Samuel is a director of the company Microimmune that produces the assay used in this study.

\section{REFERENCES}

1. Gay NJ, et al. Improving sensitivity of oral fluid testing in IgG prevalence studies: application of mixture models to a rubella antibody survey. Epidemiology and Infection 2003; 130: 285-291.

2. Vyse AJ, Cohen BJ, Ramsay ME. A comparison of oral fluid collection devices for use in the surveillance of virus diseases in children. Public Health 2001; 115: 201-207.

3. Nokes DJ, et al. Has oral fluid the potential to replace serum for the evaluation of population immunity levels? A study of measles, rubella and hepatitis B in rural Ethiopia. Bulletin of the World Health Organization 2001; 79: 588-595.

4. Nokes DJ, et al. An evaluation of oral-fluid collection devices for the determination of rubella antibody status in a rural Ethiopian community. Transactions of the Royal Socity of Tropical Medicine and Hygiene 1998; 92: 679-685.

5. Nokes DJ, et al. A comparison of oral fluid and serum for the detection of rubella-specific antibodies in a community study in Addis Ababa, Ethiopia. Tropical Medicine \& International Health 1998; 3: 258-267.

6. Vyse AJ, et al. Detection of antibody to Epstein-Barr virus viral capsid antigen in saliva by antibody capture radioimmunoassay. Journal of Virological Methods 1997; 63: 93-101.

7. Vyse AJ, et al. Detection of rubella virus-specific immunoglobulin $G$ in saliva by an amplification-based enzyme-linked immunosorbent assay using monoclonal antibody to fluorescein isothiocyanate. Journal of Clinical Microbiology 1999; 37 : 391-395.
8. Vyse AJ, et al. Interpreting serological surveys using mixture models: the seroepidemiology of measles, mumps and rubella in England and Wales at the beginning of the 21st century. Epidemiology and Infection 2006; 134: 1303-1312.

9. Nigatu W, et al. Detection of measles specific $\operatorname{IgG}$ in oral fluid using an FITC/anti-FITC IgG capture enzyme linked immunosorbent assay (GACELISA). Journal of Virological Methods 1999; 83: 135-44.

10. Hesketh L, et al. An evaluation of nine commercial EIA kits for the detection of measles specific IgG. Journal of Virological Methods 1997; 66: 51-59.

11. Kremer JR, Muller CP. Evaluation of commercial assay detecting specific immunoglobuling in oral fluid for determining measles immunity in vaccinees. Clinical and Diagnostic Laboratory Immunology 2005; 12: 668-670.

12. Mossong $\mathbf{J}$, et al. Modeling the impact of subclinical measles transmission in vaccinated populations with waning immunity. American Journal of Epidemiology $1999 ; 150$ : 1238-1249.

13. CDC. Progress in measles control - Kenya 2002-2007. Morbidity and Mortality Weekly Report 2007; 56: 969-972.

14. Kenya National Bureau of Statistics. Population distribution by province/district and sex: 1979-1999 Censuses, 1999.

15. Cutts FT, et al. Prevalence of measles antibody among children under 15 years of age in Santa Cruz, Bolivia: implications for vaccination strategies. Transactions of the Royal Socity of Tropical Medicine and Hygiene 1995; 89: 119-122.

16. Cox MJ, et al. Measles antibody levels in a vaccinated population in Brazil. Transactions of the Royal Socity of Tropical Medicine and Hygiene 1998; 92: 227-230.

17. Enquselassie F, et al. Seroepidemiology of measles in Addis Ababa, Ethiopia: implications for control through vaccination. Epidemiology and Infection 2003; 130: $507-519$.

18. WHO. Vaccine Preventable Disease unit of WHO/ African Region, Vaccine Preventable Diseases Bulletin WHO/AFRO. AFRO EPI-Newsletter, October, 2001(016).

19. WHO. Report on the 1st Consultation of the Technical Advisory Group on Measles and Rubella Control in the African Region. Harare, Zimbabwe: World Health Organisation Regional Office for Africa, 2005.

20. CDC. Effects of measles-control activities - African region, 1999-2005. Morbidity and Mortality Weekly Report 2006; 55: 1017-1021. 